\title{
Computing and Stability in Cortical Networks
}

\author{
Peter E. Latham \\ pel@gatsby.ucl.ac.uk
}

Sheila Nirenberg

sheilan@ucla.edu

Department of Neurobiology, University of California at Los Angeles,

Los Angeles, CA 90095-1763, U.S.A.

Cortical neurons are predominantly excitatory and highly interconnected. In spite of this, the cortex is remarkably stable: normal brains do not exhibit the kind of runaway excitation one might expect of such a system. How does the cortex maintain stability in the face of this massive excitatory feedback? More importantly, how does it do so during computations, which necessarily involve elevated firing rates? Here we address these questions in the context of attractor networks-networks that exhibit multiple stable states, or memories. We find that such networks can be stabilized at the relatively low firing rates observed in vivo if two conditions are met: (1) the background state, where all neurons are firing at low rates, is inhibition dominated, and (2) the fraction of neurons involved in a memory is above some threshold, so that there is sufficient coupling between the memory neurons and the background. This allows "dynamical stabilization" of the attractors, meaning feedback from the pool of background neurons stabilizes what would otherwise be an unstable state. We suggest that dynamical stabilization may be a strategy used for a broad range of computations, not just those involving attractors.

\section{Introduction}

Attractor networks-networks that exhibit multiple stable states-have served as a key theoretical model for several important computations, including associative memory (Hopfield, 1982, 1984), working memory (Amit \& Brunel, 1997a; Brunel \& Wang, 2001), and the vestibular-ocular reflex (Seung, 1996), and determining whether these models apply to real biological networks is an active area of experimental research (Miyashita \& Hayashi, 2000; Aksay, Gamkrelidzek, Seungk, Baker, \& Tank, 2001; Ojemann, Schoenfield-McNeill, \& Corina, 2002; Naya, Yoshida, \& Miyashita, 2003). A definitive determination, however, has been difficult, mainly because attractors cannot be observed directly; instead, inferences must be made about their existence by comparing experimental data with model prediction. 
To make these comparisons, it is necessary to have realistic models. Construction of such models has proved difficult because of what we refer to as the stability problem. The stability problem arises primarily because cortical networks are highly recurrent-a typical neuron in the cortex receives input from 5,000 to 10,000 others, most of which are nearby (Braitenberg \& Schüz, 1991). While this high connectivity undoubtedly provides immense computational power, it also has a downside: it can lead to instabilities in the form of runaway excitation. For example, even mild electrical stimulation applied periodically can eventually lead to seizures (McIntyre, Poulter, \& Gilby, 2002), and epilepsy, a sign of intrinsic instability, occurs in 0.5 to $1 \%$ of the human population (Bell \& Sander, 2001; Hauser, 1997).

The severity of the stability problem lies in the fact that recurrent connections in attractor networks have to be strong enough to allow activity in the absence of input, but not so strong that the activity can occur spontaneously. Moreover, in areas where attractor networks are thought to exist, such as prefrontal cortex (Fuster \& Alexander, 1971; Wilson, Scalaidhe, \& GoldmanRakic, 1993; Freedman, Riesenhuber, Poggio, \& Miller, 2001) and inferior temporal cortex (Fuster \& Jervey, 1982; Miyashita \& Chang, 1988), the firing rates associated with attractor states are not much higher than those associated with background activity. The two differ by only a few $\mathrm{Hz}$, with typical background rates ranging from 1 to $10 \mathrm{~Hz}$ and attractor rates ranging from 10 to $20 \mathrm{~Hz}$ (Fuster \& Alexander, 1971; Miyashita \& Chang, 1988; Nakamura $\&$ Kubota, 1995). This small difference makes a hard stability problem even harder, as there is almost no barrier preventing spontaneous jumps to attractors. Indeed, in previous attempts to build realistic models of attractor networks (Amit \& Brunel, 1997a; Wang, 1999; Brunel, 2000; Brunel \& Wang, 2001), fine-tuning of parameters was required to support attractors at the low rates seen in vivo. This was mainly because firing rates in those models were effectively set by single neuron saturation-by the fact that neurons simply cannot fire for extended periods above a maximum rate.

Here we propose a solution to the fine-tuning problem, one that allows attractors to exist at low rates over a broad range of parameters. The basic idea is to use natural interactions between the attractors and the background to limit firing rates, so that rates are set by network rather than single neuron properties. Limiting firing rates in this way may be a general computational strategy, not one used just by attractor networks. Thus, quantifying this mechanism in the context of attractor networks may serve as a general model for how cortical circuits carry out a broad range of computations while avoiding instabilities.

\section{Reduced Model System}

To understand qualitatively the properties of attractor networks, we analyze a model system in which the neurons are described by their firing rates. For simplicity, we start with a network that exhibits two stable equilibria: a 
background state, where all neurons fire at low rates, and a memory state, where a subpopulation of neurons fires at an elevated rate. Our results, however, apply to multiattractor networks-networks that exhibit multiple memories-and we will consider such networks below. The goal of the analysis in this section is to obtain a qualitative understanding of attractor networks, in particular, how they can fire at low rates without destabilizing the background. We will then use this qualitative understanding to guide network simulations of multiattractor networks with spiking neurons (containing up to 50 attractors), and use those simulations to verify the results obtained from the reduced model.

We construct our reduced model attractor network in two steps. First, we build a randomly connected network of excitatory and inhibitory neurons that fire at a few $\mathrm{Hz}$ on average. Second, we pick out a subpopulation of the excitatory neurons (which we refer to as memory neurons) and strengthen the connections among them (see Figure 1). If the parameters are chosen properly, this strengthening will produce a network in which the memory neurons can fire at either an elevated rate or the background rate, resulting in a bistable network.

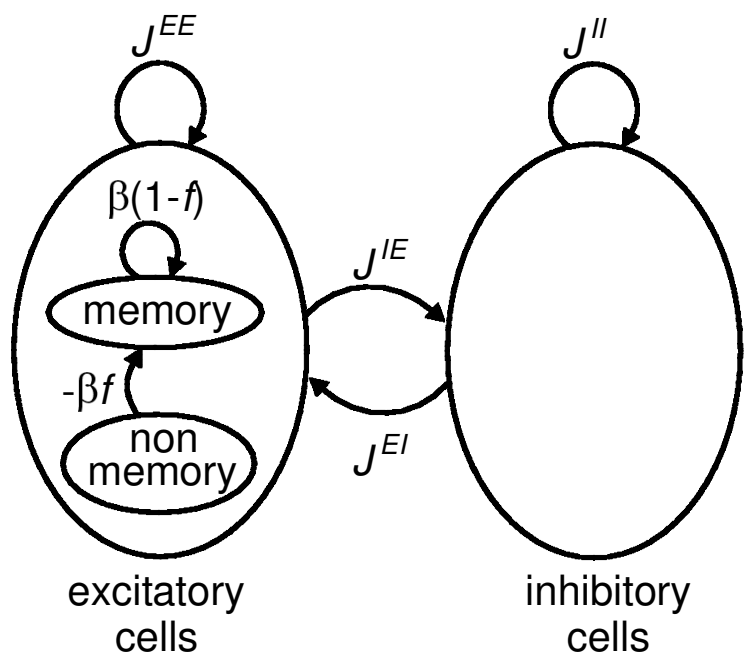

Figure 1: Schematic of network architecture. Excitatory and inhibitory neurons are synaptically coupled via the connectivity matrix $J$. The inhibitory neurons are homogeneous; the excitatory neurons break into two populations, memory and nonmemory. The coupling among memory neurons is higher than among nonmemory neurons by a factor of $\beta(1-f)$; the coupling from nonmemory to memory neurons is lower by a factor of $\beta f$. The memory neurons make up a fraction $f$ of the excitatory population, so the decrease in coupling from nonmemory to memory neurons ensures that the total synaptic drive to both memory and nonmemory neurons is the same. 
To analyze the stability and computational properties of this network, we assume that, in equilibrium, the firing rate of a neuron is a function of only the firing rates of the neurons presynaptic to it. Then, near equilibrium, we expect Wilson and Cowan-like equations (Wilson \& Cowan, 1972) in which the firing rates obey first-order dynamics. Augmenting the standard Wilson and Cowan equations to allow increased connectivity among a subpopulation of neurons (see Figure 1 and appendix A), we have

$$
\begin{aligned}
\tau_{E} \frac{d v_{E i}}{d t}+v_{E i} & =\phi_{E}\left(J^{E E} v_{E}-J^{E I} v_{I}+\frac{\beta}{N_{E} f(1-f)} \sum_{j} \xi_{i}\left(\xi_{j}-f\right) v_{E j}\right) \\
\tau_{I} \frac{d v_{I}}{d t}+v_{I} & =\phi_{I}\left(J^{I E} v_{E}-J^{I I} v_{I}\right) .
\end{aligned}
$$

Here, $\tau_{E}$ and $\tau_{I}$ are the excitatory and inhibitory time constants, $v_{E i}$ is the firing rate of the $i^{\text {th }}$ excitatory neuron $\left(i=1, \ldots, N_{E}\right), v_{E}$ and $v_{I}$ are the average firing rates of the excitatory and inhibitory neurons, respectively, the Js are the average coupling coefficients among the excitatory and inhibitory populations, $\phi_{E}$ and $\phi_{I}$ are the average excitatory and inhibitory gain functions, respectively, $\beta$ is the effective strength of the coupling among the memory neurons, $N_{E}$ is the number of excitatory neurons, and $\xi$ is a random binary vector: $\xi_{i}=1$ with probability $f$ and 0 with probability $1-f$. The factor $\xi_{j}-f$ ensures postsynaptic normalization: on average, the total synaptic strength to both memory and nonmemory neurons is the same. The $\xi$-dependent term in equation 2.1a is a standard one for constructing attractor networks (Hopfield, 1982; Tsodyks \& Feigel'man, 1988; Buhmann, 1989).

The gain functions, $\phi_{E}$ and $\phi_{I}$, play a key role in this analysis. These functions, which hide all the single neuron properties, have a natural interpretation: each one corresponds to the average firing rate of a population of neurons as a function of the average firing rates of neurons presynaptic to it. They thus have standard shapes when plotted versus $v_{E}$ : they look like $f$-I curves-firing rate versus injected current (McCormick, Connors, Lighthall, \& Prince, 1985) — that have been smoothed at low firing rates (Brunel \& Sergi, 1998; Tiesinga, José, \& Sejnowski, 2000; Fourcaud \& Brunel, 2002; Brunel \& Latham, 2003). A generic gain function is shown in Figure 2. This curve does not correspond to any particular neuron or class of neurons-it is just illustrative. There are two important aspects to its shape: (1) it has a convex region, and (2) the transition from convex to concave occurs at firing rates well above 10 to $20 \mathrm{~Hz}$ on the output side (that is, the transition occurs when $\phi \gg 10-20 \mathrm{~Hz}$ ). These properties are typical of both model neurons (Brunel \& Sergi, 1998; Tiesinga et al., 2000; Fourcaud \& Brunel, 2002; Brunel \& Latham, 2003) and real neurons (McCormick et al., 1985; Chance, Abbott, \& Reyes, 2002), and in the next two sections we will see how they connect to the problem of robust, low firing-rate attractors. 




Figure 2: Generic gain function versus average excitatory firing rate, $v_{E}$. There are three distinct regimes: First, at small $v_{E}$, the mean current induced by presynaptic firing is too low to cause a postsynaptic neuron to fire, so postsynaptic activity is due to fluctuations in the current. This is the noise-dominated regime, and here $\phi$ is convex. Second, for slightly larger $v_{E}, \phi$ becomes approximately linear or slightly concave. Finally, for large enough $v_{E}$, the firing rate saturatesat around $100 \mathrm{~Hz}$ for typical cortical pyramidal cells (McCormick et al., 1985). This gain function is a schematic and does not correspond to any particular neuron model.

Although equations 2.1a and $2.1 \mathrm{~b}$ have a fairly broad range of applicability, they are not all encompassing. In particular, they leave out the possibility of instability via synchronous oscillations (Abbott \& van Vreeswijk, 1993; Gerstner \& van Hemmen, 1993; Hansel \& Mato, 2001), and they ignore the effects of higher-order moments of the firing rate (Amit \& Brunel, 1997a; Latham, 2002). We thus verify all predictions using large-scale simulations of synaptically coupled neurons.

As indicated in Figure 1, the network described by equations 2.1a and $2.1 \mathrm{~b}$ contains a preferentially connected subpopulation. We are interested in determining under what conditions the network supports two states: a "memory" state in which this subpopulation fires at elevated rate compared to the background, and a background state in which the subpopulation fires at the same rate as the background. Since it is the difference between the attractor and background firing rates that is important, we define a variable, $m$, that is proportional to this difference; for convenience, we let 
the proportionality constant be $1 /(1-f)$,

$$
m \equiv \frac{1}{1-f}\left[\frac{1}{N_{E} f} \sum_{i} \xi_{i} \nu_{E i}-\frac{1}{N_{E}} \sum_{i} v_{E i}\right] .
$$

The first expression inside the angle brackets is the average firing rate of the subpopulation; the second is the average firing rate of the whole population, $v_{E}$. Thus, the average firing rate of the subpopulation is $v_{E}+(1-f) m$, and the background state corresponds to $m=0$.

Intuitively, we expect that network dynamics should be governed by three variables: the firing rate associated with the memory state, $m$, the average excitatory firing rate, $v_{E}$, and the average inhibitory rate, $v_{I}$. In fact, if we average equations $2.1 \mathrm{a}$ and $2.1 \mathrm{~b}$ over index, $i$, we can derive differential equations for these variables. As shown in appendix $A$, the averaged equations are

$$
\begin{aligned}
\tau_{E} \frac{d m}{d t}+m= & \phi_{E}\left(J^{E E} v_{E}-J^{E I} v_{I}+\beta m\right)-\phi_{E}\left(J^{E E} v_{E}-J^{E I} v_{I}\right) \\
\tau_{E} \frac{d v_{E}}{d t}+v_{E}= & (1-f) \phi_{E}\left(J^{E E} v_{E}-J^{E I} v_{I}\right) \\
& +f \phi_{E}\left(J^{E E} v_{E}-J^{E I} v_{I}+\beta m\right) \\
\tau_{I} \frac{d v_{I}}{d t}+v_{I}= & \phi_{I}\left(J^{I E} v_{E}-J^{I I} v_{I}\right) .
\end{aligned}
$$

To simplify our analysis, we adopt the the effective response function approach of Mascaro and Amit (1999), which can be implemented by taking the limit of fast inhibition, $\tau_{I} \rightarrow 0$. The main caveat to this limit is that we may overestimate the stability of equilibria: equilibria can be stable when $\tau_{I}=0$ but unstable when $\tau_{I}$ is above some threshold (Wilson \& Cowan, 1972; van Vreeswijk \& Sompolinsky, 1996; Latham, Richmond, Nelson, \& Nirenberg, 2000a; Hansel \& Mato, 2001). With $\tau_{I}=0$, we can solve equation 2.3c for $v_{I}$ in terms of $v_{E}$. This allows us to write $v_{I}=v_{I}\left(v_{E}\right)$, where the function $v_{I}\left(v_{E}\right)$ is determined implicitly from equation $2.3 \mathrm{c}$ with $\tau_{I}=0$. Replacing $v_{I}$ with $v_{I}\left(v_{E}\right)$ in equations $2.3 \mathrm{a}$ and $2.3 \mathrm{~b}$, we find that all the $v_{E}$-dependence on the right-hand side of these equations can be lumped into the single expression, $J^{E E} v_{E}-J^{E I} v_{I}\left(v_{E}\right)$. We denote this $-\gamma\left(v_{E}\right)$, so that

$$
\gamma\left(v_{E}\right) \equiv-J^{E E} v_{E}+J^{E I} v_{I}\left(v_{E}\right) .
$$

With $v_{I}$ eliminated, we are left with just two equations:

$$
\begin{aligned}
\tau_{E} \frac{d m}{d t}+m & =\phi_{E}\left(-\gamma\left(v_{E}\right)+\beta m\right)-\phi_{E}\left(-\gamma\left(v_{E}\right)\right) \equiv \Delta \phi_{E}\left(\nu_{E}, m\right) \\
\tau_{E} \frac{d \nu_{E}}{d t}+v_{E} & =\phi_{E}\left(-\gamma\left(v_{E}\right)\right)+f \Delta \phi_{E}\left(v_{E}, m\right) .
\end{aligned}
$$


These equations are similar to ones derived previously by Brunel and colleagues (Amit \& Brunel, 1997a; Brunel, 2000; Brunel \& Wang, 2001).

2.1 The Sparse Coding Limit. The question we are interested in is: under what conditions do equations $2.5 \mathrm{a}$ and $2.5 \mathrm{~b}$ admit two stable solutionsone with $m=0$ (the background state) and one with $m \neq 0$ (the memory state)? Before we answer this question in general, let us consider the sparse coding limit, $f \rightarrow 0$, as this limit is relatively simple and it allows us to make contact with previous work.

When $f=0$, equations $2.5 \mathrm{a}$ and $2.5 \mathrm{~b}$ decouple. In this regime, we can solve equation $2.5 \mathrm{~b}$ for the excitatory firing rate, then solve equation $2.5 \mathrm{a}$ for $m$. Let us assume that equation $2.5 \mathrm{~b}$ has a stable solution, meaning the network admits a stable background state, and focus on equation 2.5a. This equation is best solved graphically, by plotting $\Delta \phi_{E}\left(\nu_{E}, m\right)$ versus $m$ and looking for intersections of this plot with the 45 degree line; those intersections correspond to equilibria (see Figure 3). Stability can be read off the

A

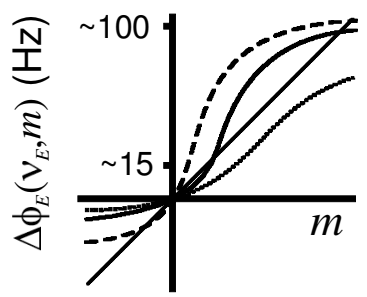

B

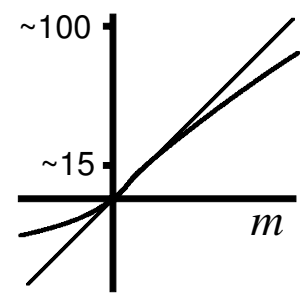

C

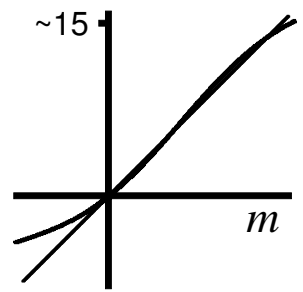

Figure 3: Gain functions and $m$-equilibria. (A). $\Delta \phi_{E}\left(v_{E}, m\right)$ versus $m$ for different values of $\beta$. Points where the curves cross the 45 degree line are equilibria. In the sparse coding limit, $f \rightarrow 0$, an equilibrium is stable if the slope of the curve is less than one and unstable otherwise. Dotted line: weak coupling (small $\beta$ ). The only equilibrium is at the background, and it is stable. Solid line: intermediate coupling. Two more equilibria have appeared. The one with intermediate $m$ is unstable; the one with large $m$ is stable. Dashed line: large coupling. The background has become destabilized, and the memory is easily activated without input. Since $\Delta \phi_{E}\left(v_{E}, m\right)$ is essentially an average single-neuron $f$-I curve, it saturates at the maximum firing rate of single neurons, $\sim 100 \mathrm{~Hz}$. Thus, the upper equilibrium occurs at high rate. (B). To reduce the firing rate of the upper equilibrium, one must operate in a very narrow parameter regime: small changes in network parameters would either rotate the curve counterclockwise, which would destabilize the background and/or move the equilibrium to high rate, or rotate it clockwise, which would eliminate the memory state. (C). Blowup of the region below $15 \mathrm{~Hz}$ in $B$. 
plots by looking at the slope: an equilibrium with slope less than 1 is stable and one with slope greater than 1 is unstable.

The number and stability of the equilibria are determined by $\beta$. If $\beta$ is small-weak coupling among the neurons in the subpopulation-there is only one equilibrium, at $m=0$, and it is stable (see Figure $3 \mathrm{~A}$, dotted line). This makes sense, as weak coupling should not have much effect on network behavior. As $\beta$ increases, so does the slope of $\Delta \phi_{E}\left(v_{E}, m\right)$, and eventually a new pair of equilibria appear (see Figure $3 \mathrm{~A}$, solid line). Of these, the one at higher firing rate (larger $m$ ) is stable, since its slope is less than 1, and the one at lower, but nonzero, rate is unstable, since its slope is greater than 1 . Finally, for large enough $\beta$, the unstable equilibrium slides past zero, at which point the equilibrium at $m=0$, and thus the background, becomes unstable (see Figure 3A, dashed line).

The intermediate $\beta$ regime, where the network can support both a memory and a stable background, is of the most interest to us. It is in this regime that the network can actually compute, in the sense that input to the network controls which state it is in (memory or background). The low and high $\beta$ regimes are not so interesting, however, at least if the goal is to construct a network that can store memories. If $\beta$ is too low, the network is stuck in the background state; if it is too high, the network can easily jump spontaneously from the background to the memory state.

As Figure 3A shows, it is not hard to build a network that can exhibit two states-so long as one is willing to allow firing rates near saturation, meaning at a healthy fraction of $100 \mathrm{~Hz}$. It is much more difficult to build a network in which the memory state exhibits low firing rates-in the 10 to $20 \mathrm{~Hz}$ range, as is observed experimentally (Fuster \& Alexander, 1971; Miyashita \& Chang, 1988; Nakamura \& Kubota, 1995). This is because low firing rates require too many bends in $\Delta \phi_{E}\left(\nu_{E}, m\right)$ in too small a firing-rate range, where "too small" is relative to the saturation firing rate of $\sim 100 \mathrm{~Hz}$ (see Figures 3B and 3C).

These qualitative arguments were quantified in networks of leaky integrate-and-fire neurons using both analytic techniques and numerical simulations (Brunel, 2000; Brunel \& Wang, 2001). For attractors that fired at about $15 \mathrm{~Hz}$, the coupling, $\beta$, among the subpopulations had to be tuned to within $1 \%$ to ensure that both the background and memories were stable. At slightly higher firing rates of 25 to $40 \mathrm{~Hz}$, the tuning was somewhat more forgiving, $3 \%$ to $5 \%$. It should be pointed out, however, that these networks were more robust to other parameters: multiple attractors were supported at reasonably low rates $(20-40 \mathrm{~Hz})$ when external input varied over a $40 \%$ range and the strengths of different receptor types (AMPA, NMDA, and GABA) were varied over range of $5 \%$ to $15 \%$.

2.2 Beyond the Sparse Coding Limit. What these results indicate is that parameters need to be finely tuned for attractor networks to exist at low rates, at least in the $f \rightarrow 0$ limit. When $f$ is finite, however, the picture 
changes, since the memories $(m)$ and background $\left(v_{E}\right)$ couple (see equation 2.5). This means that the slope of $\Delta \phi_{E}\left(v_{E}, m\right)$ with respect to $m$ is no longer the sole factor determining stability; instead, stability depends on the interaction between $m$ and $v_{E}$. Consequently, an equilibrium in which the slope of $\Delta \phi_{E}\left(\nu_{E}, m\right)$ is greater than 1 can be stable. For example, the equilibrium on the solid curve in Figure 3A that occurs at about $15 \mathrm{~Hz}$, which is unstable in the $f \rightarrow 0$ limit, could become stable when $f>0$. If this were to happen, attractors could exist robustly at low rate.

We refer to the regime in which the attractors are stable even though the slope of $\Delta \phi_{E}\left(v_{E}, m\right)$ is greater than 1 as the dynamically stabilized regime. This is because an equilibrium that would be unstable when one considers only the time evolution of the memory is dynamically stabilized by feedback from the background. Networks that operate in this regime have been investigated by Sompolinsky and colleagues (Rubin \& Sompolinsky, 1989; Golomb, Rubin, \& Sompolinsky, 1990). Although the firing rates of the attractors in those networks were low, the networks were not realistic in an important respect: they did not exhibit a background state in which all neurons fired at about the same rate; instead, the only stable states were ones in which a subpopulation of the neurons was active. Our goal here is to overcome this problem and build a network that both operates in the dynamically stabilized regime, and thus at low rates, and supports a low firing-rate background. To do this, we must evaluate the stability of the equilibria in $m-v_{E}$ space.

The network equilibria are found by setting $d m / d t$ and $d v_{E} / d t$ in equations $2.5 \mathrm{a}$ and $2.5 \mathrm{~b}$ and solving the resulting algebraic equations. With the time derivatives set to zero, the solutions to these equations are curves in $m-v_{E}$ space. These curves are referred to as nullclines, and their intersections correspond to equilibria. Constructing nullclines from the properties of $\phi_{E}$ is straightforward (Latham et al., 2000a) but tedious. We thus skip the details and simply plot them; once we have made the plots, it is relatively easy to see that they have the correct qualitative shape.

One possible set of nullclines is shown in Figure 4A, with the black curve corresponding to the $v_{E}$-nullcline (the solution to equation $2.5 \mathrm{~b}$ with $d v_{E} / d t=0$ ) and the gray curves corresponding to the $m$-nullcline (the solution to equation 2.5a with $d m / d t=0$; note that there are two pieces-a smooth curve and a vertical line). The shape of the $v_{E}$-nullcline is relatively easy to understand: it is an increasing function of $m$, reflecting the fact that as $m$ gets larger, there is more excitatory drive to the network. This can also be seen from equation $2.5 \mathrm{~b}$, where $v_{E}$ is coupled to $m$ through the term $f \Delta \phi_{E}\left(v_{E}, m\right)$, and $\Delta \phi_{E}\left(v_{E}, m\right)$ is an increasing function of $m$.

The $m$-nullcline is a little more complicated, primarily because it consists of two pieces rather than one. To understand its shape, we must reexamine Figure 3A. This figure shows three plots of $\Delta \phi_{E}\left(v_{E}, m\right)$ versus $m$. These plots correspond to three values of $\beta$; however, because $v_{E}$ as well as $\beta$ affects $\Delta \phi_{E}\left(v_{E}, m\right)$, they could just as easily have corresponded to three values of 
$v_{E}$. In fact, had we fixed $\beta$ and varied $v_{E}$, we would have obtained a set of curves qualitatively similar to the ones shown in Figure 3A. In other words, depending on the value of $v_{E}$, we would have seen three distinct regimes: (1) one equilibrium at $m=0$ (dotted line in Figure 3A), (2) one equilibrium at $m=0$ and two at $m>0$ (solid line), and (3) one equilibrium at $m=0$, one at $m<0$, and one at $m>0$ (dashed line). These three regimes are reflected in the $m$-nullcline in Figure $4 \mathrm{~A}$ : when $v_{E}$ is large, there is a single equilibrium at $m=0$ (regime 1); when $v_{E}$ is intermediate, there is an additional pair of equilibria at $m>0$ (regime 2); and when $v_{E}$ is small, one of the equilibria in that pair becomes negative (regime 3 ).

The order in which the regimes appear in Figure $4 \mathrm{~A}-$ one equilibrium at zero when $v_{E}$ is large, two positive ones when $v_{E}$ is slightly smaller,

A

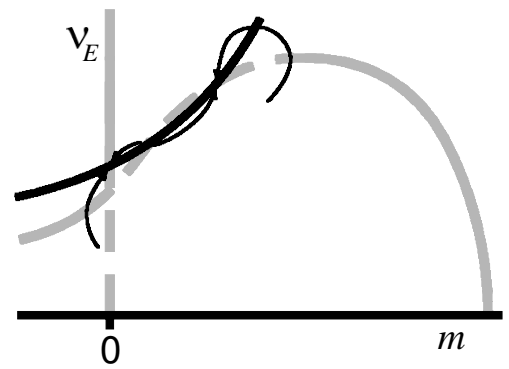

C



B

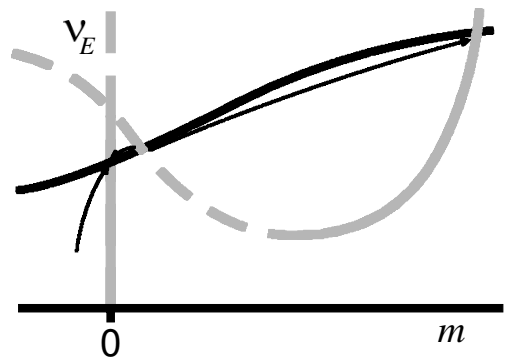

D



Figure 4: Nullclines in various parameter regimes. The gray curve, including the vertical line, is the nullcline for equation 2.5a; the black curve is the nullcline for equation $2.5 \mathrm{~b}$. The solid branches of the gray curves are stable at fixed $v_{E}$; the dashed branches are unstable at fixed $v_{E}$. The black arrows indicate typical trajectories; they are derived from equation 2.5. (A) $\Delta \phi_{E}\left(v_{E}, m\right)$ is a decreasing function of $\nu_{E} ; f$ is reasonably large. (B) $\Delta \phi_{E}\left(\nu_{E}, m\right)$ is an increasing function of $\nu_{E} ; f$ is reasonably large. (C) $\Delta \phi_{E}\left(v_{E}, m\right)$ is a decreasing function of $\nu_{E} ; f \ll 1$. (D) $\Delta \phi_{E}\left(v_{E}, m\right)$ is an increasing function of $v_{E} ; f \ll 1$. 
and a negative one when $v_{E}$ is smaller still—corresponds to a particular dependence of $\Delta \phi_{E}\left(v_{E}, m\right)$ on $v_{E}$. Examining Figure $3 \mathrm{~A}$, we see that this progression corresponds to a dependence in which $\Delta \phi_{E}\left(\nu_{E}, m\right)$ decreases as $v_{E}$ increases. Thus, for Figure $4 \mathrm{~A}$ to correctly describe the $m$-nullcline, the network parameters must be such that $\Delta \phi_{E}\left(v_{E}, m\right)$ is a decreasing function of $v_{E}$. Below, we derive explicit conditions under which this happens. First, however, we examine its consequences.

The black and gray nullclines in Figure 4 intersect in three places, and thus exhibit three equilibria. One is at $m=0$, and two are at $m>0$. The equilibrium at $m=0$ corresponds to the background state; the other two are candidates for memory states. To determine which are stable, we have plotted a few typical trajectories (black arrows), which are derived from equation 2.5. These trajectories tell us that the stable equilibria occur at $m=0$ and at the right-most intersection. Importantly, the stable equilibrium at $m>0$ occurs on the unstable branch of the $m$-nullcline, in the dynamically stabilized regime. (We can tell that this branch is unstable because at fixed $v_{E}$, the trajectories point away from it; flow is to the right below the $m$-nullcline and to the left above it. The $v_{E}$-nullcline, on the other hand, consists of one stable branch, since flow is toward it at fixed $m$. This reflects the fact that the background is always stable at fixed $m$, independent of the firing rate of the memory state.)

The unstable branches, which are drawn with dashed lines, correspond to points where the slope of $\Delta \phi_{E}\left(v_{E}, m\right)$ is greater than one. That the memory state lives on the unstable branch of the $m$-nullcline is important, because the unstable branch corresponds to the intermediate equilibrium shown in Figure $3 \mathrm{~A}$ and can thus occur at low firing rate. All previous work in realistic networks that we know of (Amit \& Brunel, 1997a; Brunel, 2000; Brunel \& Wang, 2001) puts the memory state on the stable branch of the $m$-nullclinethe part with slope less than one. The stable branch corresponds to the upper equilibrium in Figure 3A, and so tends to occur at high firing rate-at some reasonable fraction of the saturation firing rate for single neurons.

It may seem counterintuitive to have a stable equilibrium on the unstable branch, but it is well known that this can happen (Rinzel \& Ermentrout, 1989). The only caveat is that there is no guarantee of stability: the equilibrium may become unstable via a Hopf bifurcation (Marsden \& McCracken, 1976), leading to oscillations. In fact, oscillations were occasionally observed-mainly near or above the stability boundary in Figure 7 . The fact that the network did not oscillate in most of the parameter regime explored was, we believe, because the background is strongly stable (there is strong attraction to the $v_{E}$-nullcline in Figure 4).

What this analysis shows is that two conditions are necessary for building an attractor network in which the memory states occur on the unstable branch of the $m$-nullcline, and thus fire at low rates: $\Delta \phi_{E}\left(v_{E}, m\right)$ must be a decreasing function of $v_{E}$, and the fraction, $f$, of neurons involved in a memory must be large enough to give the $v_{E}$-nullcline significant curvature. 
What happens if either of these conditions is violated? Figure $4 \mathrm{~B}$ shows the nullclines when $\Delta \phi_{E}\left(v_{E}, m\right)$ is an increasing function of $v_{E}$; in this regime, the $m$-nullcline turns upside down. A low-firing-rate equilibrium still exists, but it is unstable, as indicated by the black arrows. The stable memory state in this regime is at high firing rate, near single-neuron saturation-too high to be consistent with experiment. Figures $4 \mathrm{C}$ and $4 \mathrm{D}$ show nullclines when $f \ll 1$. Again, the only stable memory states are near saturation, and thus at high rate.

Is the condition that $\Delta \phi_{E}\left(v_{E}, m\right)$ be a decreasing function of $v_{E}$ satisfied for realistic networks? In other words, is it reasonable to expect $\partial \Delta \phi_{E}\left(v_{E}, m\right) / \partial v_{E}<0$ ? To answer this, we use equation 2.5 a to write

$$
\frac{\partial \Delta \phi_{E}\left(v_{E}, m\right)}{\partial v_{E}}=-\gamma^{\prime}\left(v_{E}\right)\left[\phi_{E}^{\prime}\left(-\gamma\left(v_{E}\right)+\beta m\right)-\phi_{E}^{\prime}\left(-\gamma\left(v_{E}\right)\right)\right],
$$

where a prime after a function denotes a derivative with respect to its argument. The term in brackets on the right-hand side of equation 2.6 is typically positive for a memory lying on the unstable branch of the $m$-nullcline; this is because the unstable branch corresponds to the intermediate equilibrium in Figure $3 \mathrm{~A}$, where $\phi_{E}$ is generally convex. Thus, the sign of $\partial \Delta \phi_{E}\left(v_{E}, m\right) / \partial v_{E}$ is determined solely by the sign of $\gamma^{\prime}\left(v_{E}\right)$. For typical cortical networks, it is likely that $\gamma^{\prime}\left(\nu_{E}\right)>0$. That is because cortical networks operate in the highgain regime, in which one excitatory action potential is capable of causing more than one excitatory action potential somewhere else in the network (Abeles, 1991; Matsumura, Chen, Sawaguchi, Kubota, \& Fetz, 1996). The only way to stabilize such a system is to provide strong feedback from excitatory to inhibitory neurons, so that the inhibitory response to small increases in excitation dominates over the excitation (van Vreeswijk \& Sompolinsky, 1996; Amit \& Brunel, 1997b; Brunel \& Wang, 2001). In terms of our network variables, this means that $d\left(J^{E I} \nu_{I}\left(\nu_{E}\right)\right) / d \nu_{E}$ must be greater than $d\left(J^{E E} v_{E}\right) / d v_{E}$, which implies, via equation 2.4 , that $\gamma^{\prime}\left(v_{E}\right)>0$. Thus, the condition $\partial \Delta \phi_{E}\left(v_{E}, m\right) / \partial v_{E}<0$ is naturally satisfied in cortical networks.

Finally, we point out that a necessary condition for a low-firing-rate memory and a stable background state is that the $v_{E}$-nullcline intersect the $m=0$ line above the exchange-of-stability point (the point where the two branches of the $m$-nullcline intersect), and then intersect twice more on the unstable branch of the $m$-nullcline. A sufficient condition for this to happen is that the slope of the $v_{E}$-nullcline is less than the slope of the $m$-nullcline at $m=0$. If that condition is satisfied, then $\beta$ can be increased until the $v_{E}$-nullcline is sufficiently far above the exchange-of-stability point to guarantee two intersections on the unstable branch.

The slopes of the two nullclines, which can be determined by implicitly differentiating equations $2.5 \mathrm{a}$ and $2.5 \mathrm{~b}$, are given by

$$
\left.\frac{d \nu_{E}}{d m}\right|_{m-\text { nullcline }}=\frac{1}{\gamma^{\prime}} \frac{\beta \phi_{E}^{\prime}(-\gamma+\beta m)-1}{\phi_{E}^{\prime}(-\gamma+\beta m)-\phi_{E}^{\prime}(-\gamma)}
$$




$$
\left.\frac{d \nu_{E}}{d m}\right|_{\nu_{E}-\text { nullcline }}=\frac{\beta f \phi_{E}^{\prime}(-\gamma+\beta m)}{1+\gamma^{\prime}\left[(1-f) \phi_{E}^{\prime}(-\gamma)+f \phi_{E}^{\prime}(-\gamma+\beta m)\right]} .
$$

To calculate the slope of the $m$-nullcline at $m=0$, it is necessary to take the $m \rightarrow 0$ limit of equation 2.7a; that will ensure that we do not pick up the vertical piece of the $m$-nullcline, which has infinite slope. Using the fact that $\beta \phi_{E}^{\prime}(-\gamma+\beta m)=1$ at the exchange-of-stability point and Taylor expanding the numerator and denominator in equation 2.7a around $m=0$, we see that the slope of the $m$-nullcline is equal to $\beta / \gamma^{\prime}$ at $m=0$. A simple calculation then tells us that the slope of the $v_{E}$-nullcline at $m=0$ is a factor of $f /\left(1+1 / \gamma^{\prime} \phi_{E}^{\prime}\right)$ smaller. This ensures that for some range of $\beta$, the nullclines will exhibit the set of equilibria shown in Figure $4 \mathrm{C}$.

We can now use Figure 4 to understand why the two features listed in the beginning of section 2 (convexity over some range and transition to concavity at firing rates well above 10-20 Hz) are necessary if attractor networks are to exhibit robust, low-firing-rate equilibria in the dynamically stabilized regime. First, if gain functions are not convex over some finite range of $m$, then they will intersect the 45 degree line with a slope that is less than 1 (disregarding the trivial intersection at $m=0$ ), which eliminates the possibility of operating in the dynamically stabilized regime (see Figure 5). Second, if the transition from a convex to a concave gain function occurs at an output rate that is less than, say, $20 \mathrm{~Hz}$, then it would be possible for the equilibria in Figures $4 \mathrm{~B}$ to $4 \mathrm{D}$ to occur at firing rates less than $20 \mathrm{~Hz}$, which would imply that a stable, low-firing-rate equilibrium can exist on the stable branch of the $m$-nullcline, and thus not in the dynamically stabilized regime.



Figure 5: Concave gain functions. When the gain functions have no convex region, they intersect the 45 degree line (marked with arrows) with slope less than one, indicating that the network operates on the stable branch of the $m$ nullcline. Thus, the only way for the network to operate in the dynamically stabilized regime is for the gain functions to be convex for some range of $m$ (see Figure 3). 
The above analysis tells us what is possible in principle; to see what is possible in practice, and to determine whether attractor networks can operate at low rates in a reasonably large parameter range, we now turn to simulations.

\section{Simulations}

To verify the reduced model described above and to determine the range of parameters that supports multiple attractors, we performed simulations with a large network of synaptically coupled, spiking neurons. Network connectivity was based on the firing-rate model given in equation 2.1, with two enhancements to make it more realistic. First, we used random, sparse background connectivity rather than uniform, all-all connectivity. Second, we allowed multiple attractors - multiple memories - rather than just a single memory. To implement multiple memories, we included in the connectivity matrix a term proportional to $\beta \sum_{\mu=1}^{p} \xi_{i}^{\mu}\left(\xi_{j}^{\mu}-f\right)$, where $p$ is the number of memories and the $\xi^{\mu}$ are uncorrelated, random binary vectors with a fraction $f$ of their components equal to one (Hopfield, 1982; Tsodyks \& Feigel'man, 1988; Buhmann, 1989). This term is a natural extension of the one given in equation 2.1a. A detailed description of the network is provided in appendix $B$.

We are interested not only in whether the network can exhibit memories at low rates, but also if it can do so without fine-tuning parameters. Ideally, we would like to explore the whole parameter space. However, there are 17 parameters (see Table 1 in appendix B), making this prohibitively timeconsuming. Instead, we chose a network with reasonable parameters (e.g., synaptic and membrane time constants and PSP size; Table 1), and then varied two parameters over a broad range. The ones we varied were $V_{P S P_{E E}}$, the excitatory-to-excitatory EPSP (excitatory postsynaptic potential) size, and $\beta$, the increase in EPSP size among the neurons contained in each of the $p$ memories. At a range of points in this two-dimensional space, we checked how many memories could be embedded out of the $p$ memories we attempted to embed and whether any memories were spontaneously activated.

A run-a simulation at a particular set of parameters-lasted 12 seconds. The first 5 seconds consisted of background activity, in which the neurons fired at low average rate. At 5 seconds, all neurons in one of the memories received a $100 \mathrm{~ms}$ barrage of EPSPs. After 2 seconds, the same neurons received a second $100 \mathrm{~ms}$ barrage, this time of inhibitory postsynaptic potentials. The network then ran for another 5 seconds at background.

A successful run-one in which the desired memory was activated and deactivated at the right times, and no other memories were spontaneously activated-is shown in Figure 6A. Note that the firing rate during a memory is relatively low, about $14 \mathrm{~Hz}$. Thus, for at least one set of parameters, an attractor can exist at low rate. 




Figure 6: Simulations in which a set of memory neurons (black line) was activated at $t=5$ seconds (first vertical dashed line) and deactivated at $t=7$ seconds (second vertical dashed line). The gray line in all plots is the background firing rate. (A) The activated memory is successfully embedded for the full 2 seconds. (B) The activated memory lasts for only 1.5 seconds, indicating that the attractor is not stable. (C) A spurious memory (dashed line) is spontaneously activated, indicating that the background is not stable. Parameters are given in Table 1, with $V_{P S P_{E E}}=0.48 \mathrm{mV}, \beta=0.21 \mathrm{mV}$, and $f=0.1$. Onset times for the memories were 50 to $100 \mathrm{~ms}$, consistent with what is observed in vivo (Wilson et al., 1993; Tomita, Ohbayashi, Nakahara, Hasegawa, \& Miyashita, 1999; Naya et al., 2003). 
A



Figure 7: Summary of simulations. (A) Number of memories successfully embedded out of 50, the number attempted. (B) Average firing rate of memory neurons. The black line in both plots is the stability boundary: below it, the background is stable, meaning no spurious memories were activated; above it, the background is unstable. For EPSPs ranging from 0.2 to $0.5 \mathrm{mV}$, the network supports multiple attractors and a stable background for values of $\beta$ varying over a $15 \%$ to $25 \%$ range. Scale bar is shown to the right. Parameters are given in Table 1, with $f=0.1$.

Not all runs were successful, of course. Two things can go wrong: the desired memory might not stay active for the whole 2 seconds (see Figure 6B), or a spurious memory might become spontaneously active (see Figure 6C). To determine whether a particular set of parameters can support multiple memories without any of them becoming unstable, we activated and deactivated, one at a time, each of the $p$ memories. A particular memory was considered to be successfully embedded if it stayed active for the requisite 2 seconds, and a particular set of parameters was considered to be stable if no spurious memories were activated in any of the $p$ runs.

The results of simulations with $p=50$ are summarized in Figure 7 . Figure 7A shows the number of memories that were successfully embedded versus $V_{P S P_{E E}}$ and $\beta$. The black line in this figure is the stability boundary: the background state is stable in the region below it, meaning no spurious memories were activated (see Figure 6A); it is unstable in the region above it, meaning at least one spurious memory was activated (see Figure 6C). Figure $7 \mathrm{~B}$ shows the average firing rate of the memory neurons for those memories that were successfully embedded. As predicted by the reduced model, the firing rate is uniformly low, never exceeding $15 \mathrm{~Hz}$ in the stable regime. The background rate (not shown) was $\sim 0.1$ to $0.2 \mathrm{~Hz}$, lower than what is seen in vivo. For these network parameters, 50 memories was close to capacity: increasing $p$ to 75 drastically reduced the size of the stable regime. 
Consistent with the analysis of the reduced model, the parameter regime that supports multiple memories and a stable background is large: for EPSPs ranging from 0.2 to $0.5 \mathrm{mV}$, the region with multiple ( $>45$ ) memories extended about $0.04 \mathrm{mV}$ below the stability boundary. If we consider the dynamic range of $\beta$ to lie between zero and the stability boundary, then this corresponds to a parameter range for $\beta$ of $15 \%$ to $25 \%$.

Although the low firing rates of the memory neurons and the large parameter regime that supports multiple memories are consistent with the reduced model introduced above, they are not a direct confirmation of it. What we would like to know is whether the equilibria we observed in the simulations really do lie on the unstable branch of the $m$-nullcline, as in Figure $4 \mathrm{~A}$, or whether they in fact lie on the stable one, as in Figures $4 \mathrm{~B}$ to $4 \mathrm{D}$. One way to find out is to manipulate the nullclines, and thus the equilibrium firing rates, and then check to see if the firing rates change as predicted by the reduced model.

A convenient manipulation is one in which the $v_{E}$-nullcline is raised or lowered while the $m$-nullcline remains fixed. This is because raising the $v_{E}{ }^{-}$ nullcline lowers the average firing rate during the activation of a memory only when the equilibrium is on the unstable branch of the $m$-nullcline (see Figure $4 \mathrm{~A}$ and the inset in Figure 8); if the equilibrium is on the stable branch, raising the $v_{E}$-nullcline increases the average firing rate (see Figures 4B-4D). Examining equation 2.5, we see that the $v_{E}$-nullcline can be raised without affecting the $m$-nullcline by increasing $f$, the fraction of neurons involved in a memory. The prediction from the reduced model, then, is that the background firing rate during the activation of a memory should decrease as $f$ increases. This prediction is verified in Figure 8 , thus providing strong evidence that the reduced model really does explain the simulations and that the simulations operate in a regime corresponding to the nullclines shown in Figure $4 \mathrm{~A}$. Note also that the range of $f$ that supports stable memories is large, $\sim 30 \%$, providing further evidence for the robustness of the network.

\section{Discussion}

The question we address in this article is: how can cortical networks, which are highly interconnected and dominated by excitatory neurons, carry out computations and yet maintain stability? We answered this question in the context of attractor networks-networks that support multiple stable states, or memories. We chose attractor networks for two reasons. First, they are thought to underlie several computations in the brain, including associative memory (Hopfield, 1982, 1984), working memory (Amit \& Brunel, 1997a; Brunel \& Wang, 2001), and the vestibular-ocular reflex (Seung, 1996). Consequently, they are an active area of experimental research (Miyashita \& Hayashi, 2000; Aksay et al., 2001; Ojemann et al., 2002; Naya et al., 2003). Second, the stability problem in attractor networks is especially severe, so 


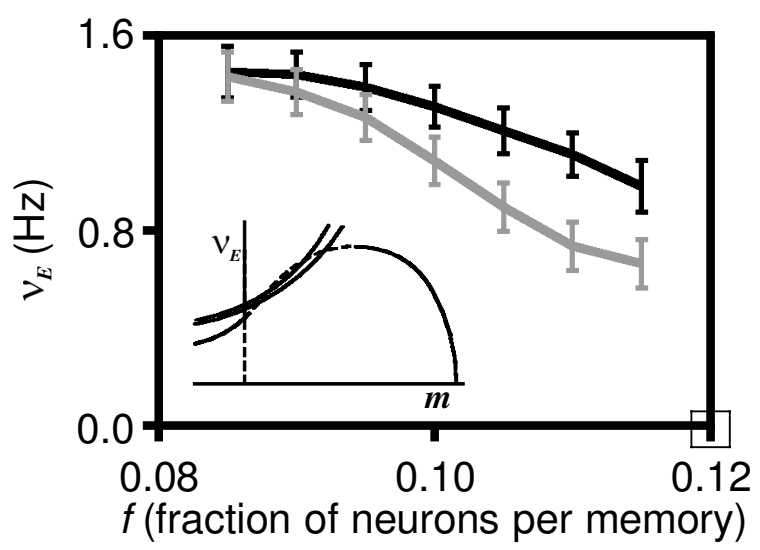

Figure 8: Average firing rate during the activation of a memory, $v_{E}$, as a function of the fraction of neurons involved in a memory, $f$, for two parameter sets. Black curve: $V_{P S P_{E E}}=0.40 \mathrm{mV}, \beta=0.18 \mathrm{mV}$; gray curve: $V_{P S P_{E E}}=0.20 \mathrm{mV}, \beta=0.21$ $\mathrm{mV}$; other parameters are given in Table 1. Averages are over all 50 memories; error bars are standard deviations. As indicated in the inset, the prediction of our model is that the firing rate, $v_{E}$, should drop as $f$ increases and drives the $v_{E}$-nullcline up (the upper $v_{E}$-nullcline corresponds to larger $f$; see the text). This prediction is verified by the decreasing firing rate versus $f$.

if we can solve this problem for attractor networks, we should be able to solve it for networks implementing other kinds of computations.

In previous models of realistic attractors networks, the strategy was to operate on the stable branch of the $m$-nullcline (Amit \& Brunel, 1997a; Wang, 1999; Brunel, 2000; Brunel \& Wang, 2001), where firing rates are set essentially by the saturation rates of single neurons (see Figure $4 \mathrm{C}$ ). Those rates are $\sim 100 \mathrm{~Hz}$, making the experimentally observed 10 to $20 \mathrm{~Hz}$ (Fuster \& Alexander, 1971; Miyashita \& Chang, 1988; Nakamura \& Kubota, 1995) hard to reach without fine-tuning parameters. Here we showed, using a reduced, two-variable model and simulations with large networks of spiking neurons, that attractor networks can operate on the unstable branch of the $m$-nullcline, and thus at low rates.

There are two key components to this model. The first is strong coupling between excitatory and inhibitory cells. This coupling, which is essential in networks with strong recurrent excitatory connections like those found in the cortex, means that any upward fluctuation in excitatory firing rate is matched by a larger upward fluctuation in inhibitory rate. (Here "larger" has a technical definition: it means that $\gamma^{\prime}\left(v_{E}\right)>0$; see equations 2.4 and 2.6.) This makes the background state effectively inhibitory, a fact that is somewhat counterintuitive but extremely valuable, as it allows the background to act as a stabilizing pool. The second component is strong coupling between 
the attractors and the background state. Since the background is effectively inhibitory, this coupling can dynamically stabilize what would otherwise be an unstable state, and thus allow operation on the unstable branch of the $m$-nullcline.

The approach we used, in which the network operates on the unstable branch of the $m$-nullcline, is fundamentally different from approaches in which networks operate on the stable branch. In particular, the latter require fine-tuning of network parameters to keep neurons from firing near saturation (see Figure 3C); the former does not. Importantly, the fine-tuning problem associated with operation on the stable branch cannot be eliminated simply by including effects like synaptic and spike-frequency adaption, or by adding inhibition. This is because the fine-tuning problem is associated with the structure of the nullclines in Figure 4 and the fact that neurons saturate at about $100 \mathrm{~Hz}$ (even with spike frequency adaptation taken into account), neither of which is changed by these manipulations.

To verify the predictions of the reduced model, we performed large-scale simulations with networks of spiking neurons. We found that $\sim 50$ overlapping memories could be embedded in a network of 10,000 neurons. As predicted, the firing rates of the neurons in the attractor were low, between 10 and $15 \mathrm{~Hz}$ on average-approximately what is seen in vivo. More important, the network was stable over a broad range of parameters: background EPSP amplitude could vary by $\sim 100 \%$, the connection strength among the neurons involved in a memory could vary by $\sim 25 \%$, and the fraction of neurons involved in a memory could vary by $\sim 30 \%$, all without degrading network operation (see Figures 7 and 8).

4.1 Implications for Memory Storage. An important outcome of our model is that it predicts that the fraction of neurons involved in a memory, $f$, must be above some threshold. Otherwise, the feedback between the memory and the background would be too weak to stabilize activity at low rates, as can be seen by comparing Figures $4 \mathrm{~A}$ and 4C. As shown by Gardner (1988), Tsodyks and Feigel'man (1988), Buhmann (1989), and Treves, Skaggs, and Barnes (1996), the maximum number of memories that can be embedded in a network is $\sim 0.2 \mathrm{~K} /|f \log f|$ where $K$ is the number of connections per neuron. This relation, combined with our result that $f$ must be above some minimum, means that there is a limit to the number of memories that can be stored in any one network. This precludes the possibility of increasing the number of memories by adding neurons to a network while keeping the number of neurons in a memory fixed: this manipulation would decrease $f$ and thus increase the maximum number of allowed memories, but as $f$ becomes too small, it would ultimately make the network unable to operate at low rates.

If we take $f=0.1$ (Fuster \& Jervey, 1982) and $K=5000$ (Braitenberg \& Schüz, 1991), then the maximum number of memories that can be stored in any strongly interconnected network in the brain is $\sim 4000$. This has ma- 
jor implications for how we store memories. Humans, for example, can remember significantly more than 4000 items, even within a particular category (words, for example). Thus, our model implies that memory must be highly distributed: local networks store at most thousands of items each, and during recall, those items must be dynamically linked to form a complete memory.

4.2 Biological Plausibility of Our Model. Our simulations contained garden-variety neurons and synapses-simple point neurons and fast synapses. This allowed us to cleanly separate behavior due to collective interactions from behavior due to single-neuron properties. However, it leaves open the possibility that more realistic neurons and synapses might change our findings, especially the size of the parameter range in which the network is stable. Probably the most important parameters for stability are the excitatory and inhibitory synaptic time constants, with long excitatory time constants being stabilizing and long inhibitory time constants destabilizing (Tegner, Compte, \& Wang, 2002). In our networks, we used the same time constant for both (3 ms). More realistic would be a longer inhibitory time constant (Salin \& Prince, 1996; Xiang, Huguenard, \& Prince, 1998) and a mix of long (NMDA) and short (AMPA) excitatory time constants (Andrasfalvy \& Magee, 2001). Fortunately, such a mix has an overall stabilizing effect (Wang, 1999). Thus, we expect that with more realistic synapses, the parameter regime that supports a large number of memories should, in fact, be larger than the one we found.

Because slow excitatory synapses reduce fluctuations, they may increase the number of memories that can be embedded. This is important, since the number of memories in our network, 50, was smaller than what we expect of realistic networks, and much smaller than the theoretical maximum of 2000 for our network (derived using the above formula, $0.2 K /|f \log f|$, with $K=2500$ and $f=0.1$ ).

The background firing rate in our simulations was $\sim 0.1$ to $0.2 \mathrm{~Hz}$, lower than the 1 to $10 \mathrm{~Hz}$ observed in vivo (Fuster \& Alexander, 1971; Miyashita \& Chang, 1988; Nakamura \& Kubota, 1995). These low rates can be traced to the fact that our network consisted of two pools of neurons, one near threshold and one well below threshold, with the latter pool firing at a very low rate. The below-threshold pool was needed to ensure that the average gain function, $\phi_{E}$, was convex at the background firing rate, which is necessary for memories to exist (see Figure 3). With other methods for achieving convex gain functions, such as coherent external input or nonlinearities in the current-to-firing-rate transformation (especially the nonlinearity provided by persistent neurons; Egorov, Hamam, Fransen, Hasselmo, \& Alonso, 2002), it is likely that a larger background rate could be supported. A nonlinear current-to-firing-rate transformation is especially helpful: when we included even a slight nonlinearity, the background rate increased to about $1 \mathrm{~Hz}$ and the capacity of the network increased to 100 memories (data 
not shown). Alternatively, it is possible that our network was consistent with real cortex, and the firing rates in vivo are overestimated. This could happen because experimental recordings are, by necessity, biased toward neurons that fire at detectable rates, whereas we averaged firing rates over all neurons in the network, some of which rarely fired. This possibility is strengthened by the recent finding that, based on energy considerations, average firing rates in the cortex are likely to be less than $1 \mathrm{~Hz}$ (Lennie, 2003).

4.3 Summary. These results demonstrate that dynamical stabilization can be used to embed multiple, overlapping, low-firing-rate attractors over a broad range of network parameters. This opens the door to detailed comparison between theory and experiment, and should help resolve the question of whether attractor networks really do exist in the brain. In addition, dynamical stabilization can be used for all kinds of networks, not just those involving attractors, and may turn out to be a general computational principle.

\section{Appendix A: Derivation of Reduced Model Equations}

In large neuronal networks in which neurons are firing asynchronously, it is reasonable to model neurons by their firing rates (Amit \& Brunel, 1997a). While this approach is unlikely to capture the full temporal network dynamics (Treves, 1993), it is useful for studying equilibria. Moreover, near an equilibrium, we expect the firing rates to obey first-order dynamics. Thus, a firing-rate model that uses linear summation followed by a nonlinearity would be described by the equations

$$
\begin{aligned}
\tau_{E} \frac{d v_{E i}}{d t}+v_{E i} & =\phi_{E i}\left(\sum_{j} J_{i j}^{E E} v_{E j}-\sum_{j} J_{i j}^{E I} v_{I j}\right) \\
\tau_{I} \frac{d v_{I i}}{d t}+v_{I i} & =\phi_{I i}\left(\sum_{j} J_{i j}^{I E} v_{E j}-\sum_{j} J_{i j}^{I I} v_{I j}\right),
\end{aligned}
$$

where $v_{E i}$ and $v_{I i}$ are the firing rates of individual excitatory and inhibitory neurons, respectively, $\phi_{E i}$ and $\phi_{I i}$ are their gain functions, and $J_{i j}$ determines the connection strength from neuron $j$ to neuron $i$.

The behavior of the network described by equation A.1 depends critically on connectivity. If the connectivity is purely random, then the network can be described qualitatively by the Wilson and Cowan model (Wilson \& Cowan, 1972). However, if the connectivity among a subpopulation of excitatory neurons is strengthened, as it is in this study, we need to augment the Wilson and Cowan model. Ignoring firing-rate fluctuations, an approx- 
imation that is valid qualitatively (Amit \& Brunel, 1997a; Latham, 2002), we can construct heuristically such an augmented model by letting

$$
\begin{aligned}
& J_{i j}^{E E} \rightarrow N_{E}^{-1} J^{E E}+\beta\left[N_{E} f(1-f)\right]^{-1} \xi_{i}\left(\xi_{j}-f\right) \\
& J_{i j}^{L M} \rightarrow N_{M}^{-1} J^{L M}, \quad L M=E I, I E, I I .
\end{aligned}
$$

In these expression, $N_{E}$ and $N_{I}$ are the number of excitatory and inhibitory neurons, respectively, and $\xi$ is a random binary vector: $\xi_{i}$ is 1 with probability $f$ and 0 with probability $1-f$. If we apply the replacements given in equation A.2 to equation A.1, average equation A.1b over index, $i$, define $\phi_{I}(x) \equiv N_{I}^{-1} \sum_{i} \phi_{I i}(x)$, and let $\phi_{E i} \rightarrow \phi_{E}$, then equation A.1 turns into equation 2.1. Replacing $\phi_{E i}$ with $\phi_{E}$ was done for convenience only: it simplifies the resulting equations without detracting from the main result.

To derive equations $2.3 \mathrm{a}$ and $2.3 \mathrm{~b}$ from equation 2.1 , we must average $\phi_{E}$ and $\xi_{i} \phi_{E}$ over index. To perform these averages, we first use the definition of $m$, equation 2.2, to simplify the argument of $\phi_{E}$; this definition implies that $\phi_{E}=\phi_{E}\left(J^{I E} v_{E}-J^{I I} v_{I}+\beta m \xi_{i}\right)$. We then note that for any function $F\left(\xi_{i}\right)$,

$$
\begin{aligned}
N_{E}^{-1} \sum_{i} F\left(\xi_{i}\right) & =N_{E}^{-1} \sum_{i}\left(1-\xi_{i}\right) F\left(\xi_{i}\right)+N_{E}^{-1} \sum_{i} \xi_{i} F\left(\xi_{i}\right) \\
& =N_{E}^{-1} \sum_{i}\left(1-\xi_{i}\right) F(0)+N_{E}^{-1} \sum_{i} \xi_{i} F(1) \\
& =(1-f) F(0)+f F(1) .
\end{aligned}
$$

The second line follows because $\xi_{i}$ is either 0 or 1 ; the third (which is strictly valid only in the $N_{E} \rightarrow \infty$ limit) because $\xi_{i}$ averages to $f$. Similarly,

$$
\left(f N_{E}\right)^{-1} \sum_{i} \xi_{i} F\left(\xi_{i}\right)=\left(f N_{E}\right)^{-1} \sum_{i} \xi_{i} F(1)=F(1) .
$$

Equations A.3 and A.4, along with the definition of $m$ given in equation 2.2, can be used to derive equations $2.3 \mathrm{a}$ and $2.3 \mathrm{~b}$.

\section{Appendix B: Simulation Details}

The network we simulate consists of $N_{E}$ excitatory and $N_{I}$ inhibitory quadratic integrate-and-fire neurons (Ermentrout \& Kopell, 1986; Ermentrout, 1996; Gutkin \& Ermentrout, 1998; Brunel \& Latham, 2003). The membrane potential of the $i$ th neuron, $V_{i}$, and the conductance change at neuron $i$ in response to a spike at neuron $j, s_{i j}$, evolve according to

$$
\tau \frac{d V_{i}}{d t}=\frac{\left(V_{i}-V_{r}\right)\left(V_{i}-V_{t}\right)}{V_{t}-V_{r}}+V_{0 i}-\left(V_{i}-\mathcal{E}_{E}\right) \sum_{j \in E} J_{i j} s_{i j}(t)
$$




$$
\begin{array}{r}
-\left(V_{i}-\mathcal{E}_{I}\right) \sum_{j \in I} J_{i j} s_{i j}(t) \\
\frac{d s_{i j}}{d t}=-\frac{s_{i j}}{\tau_{s}}+\sum_{l} \delta\left(t-t_{j}^{l}\right) .
\end{array}
$$

Here $\tau$ is the cell time constant, $V_{r}$ and $V_{t}$ are the nominal resting and threshold voltages, $V_{0 i}$ is the product of the applied current and the membrane resistance (in units of voltage), $J_{i j}$ is the (dimensionless) connection strength from cell $j$ to cell $i, \mathcal{E}_{E}$ and $\mathcal{E}_{I}$ are the excitatory and inhibitory reversal potentials, respectively, the notation $j \in M$ means sum over only those cells of type $M, \delta(\cdot)$ is the Dirac $\delta$-function, and $t_{j}^{l}$ is the $l$ th spike emitted by neuron $j$.

To mimic the heterogeneity seen in cortical neurons, we let $V_{o i}$, which determines the distance from resting membrane potential to threshold, have a range of values. This range is captured by the distributions

$$
\begin{aligned}
& P_{E}\left(V_{0}\right)=0.75 \frac{\exp \left[-\left(V_{0}-1.5\right)^{2} / 2(0.5)^{2}\right]}{\left[2 \pi(0.5)^{2}\right]^{1 / 2}} \\
& +0.25 \frac{\exp \left[-\left(V_{0}-3.75\right)^{2} / 2(1.0)^{2}\right]}{\left[2 \pi(1.0)^{2}\right]^{1 / 2}} \\
& P_{I}\left(V_{0}\right)=\frac{1}{4.5} \times\left\{\begin{array}{ll}
1 & 0.5<V_{0}<5.0 \\
0 & \text { otherwise }
\end{array}\right. \text {, }
\end{aligned}
$$

where $P_{E}\left(V_{0}\right)$ and $P_{I}\left(V_{0}\right)$ are the distributions for excitatory and inhibitory neurons, respectively. In the absence of synaptic drive, the distance between resting membrane potential and threshold is $\left(V_{t}-V_{r}\right)\left[1-4 V_{0} /\left(V_{t}-V_{r}\right)\right]^{1 / 2}$ (see equation B.1a). Since we use $V_{t}-V_{r}=15 \mathrm{mV}$ (see Table 1), $V_{0}=3.75$ corresponds to a resting membrane potential that is equal to threshold while $V_{0}=1.5$ corresponds to a resting membrane potential about $12 \mathrm{mV}$ below threshold. Neurons for which $V_{0}>3.75$ are endogenously active-they fire repeatedly without input.

The distribution $P_{E}\left(V_{0}\right)$ tells us that the excitatory neurons consist of two pools: one with resting membrane potential well below threshold, and one with resting membrane potential near threshold. About half the neurons in the latter pool are above threshold, and thus endogenously active. In realistic networks, this endogenous activity could be due to external input, intrinsic single-neurons properties (Latham, Richmond, Nirenberg, \& Nelson, 2000b), or a combination of the two. While endogenously active cells greatly facilitate the existence of a low-firing-rate background state (Latham et al., 2000a), they are not absolutely necessary for it (Hansel \& Mato, 2001).

A spike is emitted from neuron $j$ whenever $V_{j}$ reaches $+\infty$, at which point it is reset to $-\infty$. To attain the values $\pm \infty$ in our numerical integration, we make the change of variables $V=\left(V_{r}+V_{t}\right) / 2+\left(V_{t}-V_{r}\right) \tan \theta$ and integrate $\theta$ instead of $V$. 
The connectivity matrix, $J_{i j}$, consists of two parts. One is random, corresponding to background connectivity before learning; the other is structured, corresponding to memories. In addition, we impose sparse connectivity by making the connection probability less than 1 . We thus write

$$
J_{i j}=g\left(c_{i j}\left(W_{i j}+A_{i j}\right)\right),
$$

where $c_{i j}$ is 1 with probability $c_{\text {connect }}$ and zero otherwise, $W_{i j}$ corresponds to the background connectivity, $A_{i j}$ corresponds to the structured connectivity, and $g$ is a clipping function chosen so that $0 \leq g(x) \leq g_{\max }$; for convenience, we choose $g(x)$ to be threshold linear and truncated at $g_{\max }$ : $g(x)=\max (x, 0)-\max \left(x-g_{\max }, 0\right)$. The clipping function ensures that a particular connection strength neither violates Dale's law by falling below zero nor exceeds physiological levels by becoming too large.

The random part of the connectivity matrix, $W$, is chosen to produce realistic postsynaptic potentials. Specifically, we let

$$
W_{i j}=w_{i j} \frac{V_{P S P_{L M}}}{V_{M}},
$$

where neuron $i$ is of type $L$, neuron $j$ is of type $M(L, M=E, I), w_{i j}$ is a random variable uniformly distributed between $1-\sqrt{3} \Delta$ and $1+\sqrt{3} \Delta$ (so that its variance is $\Delta^{2}$ ), and

$$
V_{M} \equiv \frac{\mathcal{E}_{M}-V_{r}}{\left(\tau / \tau_{s}\right) \exp \left[\ln \left(\tau / \tau_{s}\right) /\left(\tau / \tau_{s}-1\right)\right]} .
$$

With this choice for the connection matrix, a neuron of type $L$ will exhibit postsynaptic potentials on the order of $V_{P S P_{L M}}$ when a neuron of type $M$ fires, assuming that $V_{0}=0$ for the neuron of type $L$ (Latham et al., 2000a).

The structured part of the connectivity matrix is the natural multimemory extension of the matrix given in equation 2.1 a, except with a slightly different normalization,

$$
A_{i j}=\frac{\beta / V_{E}}{N_{E} f(1-f)} \sum_{\mu=1}^{p} \xi_{i}^{\mu}\left(\xi_{j}^{\mu}-f\right),
$$

where $p$ is the number of memories and the $\xi^{\mu}$ are uncorrelated, random binary vectors: $\xi_{i}^{\mu}$ is 1 with probability $f$ and 0 with probability $1-f$. Only excitatory neurons participate in the memories. The factor $1 / V_{E}$ is included so that $\beta$ has units of voltage.

The parameters in the model are listed in Table 1. All were fixed throughout the simulation except for $\beta$ and $V_{P S P_{E E}}$, which were varied to explore robustness (see Figure 7), and $f$, which was 0.1 except in Figure 8, where it ranged from 0.085 to 0.115 . 
Table 1: Parameters Used in the Simulations.

\begin{tabular}{lc}
\hline Excitatory neurons & 8000 \\
Inhibitory neurons & 2000 \\
$c_{\text {connect }}$ & 0.25 \\
$\Delta$ & 0.25 \\
$\tau$ & $10 \mathrm{~ms}$ \\
$\tau_{s}$ & $3 \mathrm{~ms}$ \\
$V_{r}$ & $-65 \mathrm{mV}$ \\
$V_{t}$ & $-50 \mathrm{mV}$ \\
$\mathcal{E}_{E}$ & $0 \mathrm{mV}$ \\
$\mathcal{E}_{I}$ & $-80 \mathrm{mV}$ \\
$V_{\text {EPSP }}(\mathrm{E} \rightarrow \mathrm{E})$ & $0.2-0.8 \mathrm{mV}$ \\
$V_{\text {EPSP }}(\mathrm{E} \rightarrow \mathrm{I})$ & $1.0 \mathrm{mV}$ \\
$V_{\text {IPSP }}(\mathrm{I} \rightarrow \mathrm{E}, \mathrm{I} \rightarrow \mathrm{I})$ & $-1.5 \mathrm{mV}$ \\
$g_{\max }$ & $2.5 \mathrm{mV}$ \\
$\beta$ & $0.08-0.28 \mathrm{mV}$ \\
$p$ & 50 \\
$f$ & $0.085-0.115$ \\
Time step & $0.5 \mathrm{~ms}$ \\
\hline
\end{tabular}

\section{Acknowledgments}

We thank Nicolas Brunel and Alex Pouget for insightful comments on the manuscript. This work was supported by NIMH Grant R01 MH62447.

\section{References}

Abbott, L., \& van Vreeswijk, C. (1993). Asynchronous states in networks of pulse-coupled oscillators. Phys. Rev. E, 48, 1483-1490.

Abeles, M. (1991). Corticonics: Neural circuits of the cerebral cortex. Cambridge: Cambridge University Press.

Aksay, E., Gamkrelidzek, G., Seungk, H., Baker, R., \& Tank, D. (2001). In vivo intracellular recording and perturbation of persistent activity in a neural integrator. Nature Neurosci., 4, 184-193.

Amit, D., \& Brunel, N. (1997a). Dynamics of a recurrent network of spiking neurons before and following learning. Network, 8, 373-404.

Amit, D., \& Brunel, N. (1997b). Model of global spontaneous activity and local structured activity during delay periods in the cerebral cortex. Cereb. Cortex, 7 , 237-252.

Andrasfalvy, B., \& Magee, J. (2001). Distance-dependent increase in AMPA receptor number in the dendrites of adult hippocampal ca1 pyramidal neurons. J. Neurosci., 21, 9151-9159.

Bell, G., \& Sander, J. (2001). The epidemiology of epilepsy: The size of the problem. Seizure, 10, 306-314.

Braitenberg, V., \& Schüz, A. (1991). Anatomy of the cortex. Berlin: Springer-Verlag. 
Brunel, N. (2000). Persistent activity and the single-cell frequency-current curve in a cortical network model. Network: Computation in Neural Systems, 11, 261-280.

Brunel, N., \& P. Latham (2003). Firing rate of the noisy quadratic integrate-andfire neuron. Neural Comput., 15, 2281-2306.

Brunel, N., \& Sergi, S. (1998). Firing frequency of leaky intergrate-and-fire neurons with synaptic current dynamics. J. Theor. Biol., 195, 87-95.

Brunel, N., \& Wang, X. (2001). Effects of neuromodulation in a cortical network model of object working memory dominated by recurrent inhibition. J. Comput. Neurosci., 11, 63-85.

Buhmann, J. (1989). Oscillations and low firing rates in associative memory neural networks. Phys. Rev. A, 40, 4145-4148.

Chance, F., Abbott, L., \& Reyes, A. (2002). Gain modulation from background synaptic input. Neuron, 35, 773-782.

Egorov, A., Hamam, B., Fransen, E., Hasselmo, M., \& Alonso, A. (2002). Graded persistent activity in entorhinal cortex neurons. Nature, 420, 173-178.

Ermentrout, B. (1996). Type i membranes, phase resetting curves, and synchrony. Neural Comput., 8, 979-1001.

Ermentrout, B., \& Kopell, N. (1986). Parabolic bursting in an excitable system coupled with a slow oscillation. SIAM J. Appl. Math., 46, 233-253.

Fourcaud, N., \& Brunel, N. (2002). Dynamics of the firing probability of noisy integrate-and-fire neurons. Neural Comput., 14, 2057-2110.

Freedman, D., Riesenhuber, M., Poggio, T., \& Miller, E. (2001). Categorical representation of visual stimuli in the primate prefrontal cortex. Science, 291, 312-316.

Fuster, J., \& Alexander, G. (1971). Neuron activity related to short-term memory. Science, 173, 652-654.

Fuster, J., \& Jervey, J. (1982). Neuronal firing in the inferotemporal cortex of the monkey in a visual memory task. J. Neurosci., 2, 361-375.

Gardner, E. (1988). The space of interactions in neural network models. J. Phys. A: Math. Gen., 21, 257-270.

Gerstner, W., \& van Hemmen, L. (1993). Coherence and incoherence in a globally coupled ensemble of pulse-emitting units. Phys. Rev. Lett., 71, 312-315.

Golomb, D., Rubin, N., \& Sompolinsky, H. (1990). Willshaw model: Associative memory with sparse coding and low firing rates. Phys. Rev. A, 41, 18431854.

Gutkin, B., \& Ermentrout, B. (1998). Dynamics of membrane excitability determine interspike interval variability: A link between spike generation mechanisms and cortical spike train statistics. Neural Comput., 10, 1047-1065.

Hansel, D., \& Mato, G. (2001). Existence and stability of persistent states in large neuronal networks. Phys. Rev. Lett., 86, 4175-4178.

Hauser, W. (1997). Incidence and prevelance. In J. Engel \& T. A. Pedley (Eds.), Epilepsy: A comprehensivetextbook(Vol. 1, pp. 47-58). Philadelphia: LippincottRaven.

Hopfield, J. J. (1982). Neural networks and physical systems with emergent collective computational abilities. Proc. Natl. Acad. Sci., 79, 2554-2558. 
Hopfield, J. J. (1984). Neurons with graded responses have collective computational properties like those of two-state neurons. Proc. Natl. Acad. Sci., 81, 3088-3092.

Latham, P. (2002). Associative memory in realistic neuronal networks. In T. G. Dietterich, S. Becker, \& Z. Ghahramani (Eds.), Advances in Neural Information Processing Systems, 14. Cambridge MA: MIT Press.

Latham, P., Richmond, B., Nelson, P., \& Nirenberg, S. (2000a). Intrinsic dynamics in neuronal networks. I. Theory. J. Neurophysiol., 83, 808-827.

Latham, P., Richmond, B., Nirenberg, S., \& Nelson, P. (2000b). Intrinsic dynamics in neuronal networks. II. Experiment. J. Neurophysiol., 83, 828-835.

Lennie, P. (2003). The cost of cortical computation. Curr. Biol., 13, 493-497.

Marsden, J., \& McCracken, M. (1976). The Hopf bifurcation and its applications. Berlin: Springer-Verlag.

Mascaro, M., \& Amit, D. (1999). Effective neural response function for collective population states. Network, 10, 351-373.

Matsumura, M., Chen, D., Sawaguchi, T., Kubota, K., \& Fetz, E. (1996). Synaptic interactions between primate precentral cortex neurons revealed by spiketriggered averaging of intracellular membrane potentials in vivo. J. Neurosci., 16, 7757-7767.

McCormick, D., Connors, B., Lighthall, J., \& Prince, D. (1985). Comparative electrophysiology of pyramidal and sparsely spiny stellate neurons of the neocortex. J. Neurophysiol., 54, 782-806.

McIntyre, D., Poulter, M., \& Gilby, K. (2002). Kindling: Some old and some new. Epilepsy Res., 50, 79-92.

Miyashita, Y., \& Chang, H. (1988). Neuronal correlate of pictorial short-term memory in the primate temporal cortex. Nature, 331, 68-70.

Miyashita, Y., \& Hayashi, T. (2000). Neural representation of visual objects: Encoding and top-down activation. Curr. Opin. Neurobiol., 10, 187194.

Nakamura, K., \& Kubota, K. (1995). Mnemonic firing of neurons in the monkey temporal pole during a visual recognition memory task. J. Neurophysiol., 74, 162-178.

Naya, Y., Yoshida, M., \& Miyashita, Y. (2003). Forward processing of long-term associative memory in monkey inferotemporal cortex. J. Neurosci., 23, 28612871.

Ojemann, G., Schoenfield-McNeill, J., \& Corina, D. (2002). Anatomic subdivisions in human temporal cortical neuronal activity related to recent verbal memory. Nature Neurosci., 5, 64-71.

Rinzel, J., \& Ermentrout, G. (1989). Models for excitable cells and networks. In C. Koch \& I. Segev (Eds.), Methods in neuronal modeling: from synapses to networks (pp. 137-173). Cambridge, MA: MIT Press.

Rubin, N., \& Sompolinsky, H. (1989). Neural networks with low local firing rates. Europhys. Lett., 10, 465-470.

Salin, P. A., \& Prince, D. A. (1996). Spontaneous $\mathrm{GABA}_{\mathrm{A}}$ receptor-mediated inhibitory currents in adult rat somatosensory cortex. J. Neurophysiol., 75, 1573-1588. 
Seung, H. (1996). How the brain keeps the eyes still. Proc. Natl. Acad. Sci., 93, 13339-13344.

Tegner, J., Compte, A., \& Wang, X. (2002). The dynamical stability of reverberatory neural circuits. Biol. Cybern., 87, 471-481.

Tiesinga, P., José, J., \& Sejnowski, T. (2000). Comparison of current-driven and conductance-driven neocortical model neurons with hodgkin-huxley voltage-gated channels. Phys. Rev. E, 62, 8413-8419.

Tomita, H., Ohbayashi, M., Nakahara, K., Hasegawa, I., \& Miyashita, Y. (1999). Top-down signal from prefrontal cortex in executive control of memory retrieval. Nature, 401, 699-703.

Treves, A. (1993). Mean-field analysis of neuronal spike dynamics. Network, 4 , 259-284.

Treves, A., Skaggs, W., \& Barnes, C. (1996). How much of the hippocampus can be explained by functional constraints? Hippocampus, 6, 666-674.

Tsodyks, M., \& Feigel'man, M. (1988). The enhanced storage capacity in neural networks with low activity level. Europhys. Lett., 6, 101-105.

van Vreeswijk, C., \& Sompolinsky, H. (1996). Chaos in neuronal networks with balanced excitatory and inhibitory activity. Science, 274, 1724-1726.

Wang, X. (1999). Synaptic basis of cortical persistent activity: The importance of NMDA receptors to working memory. J. Neurosci., 19, 9587-9603.

Wilson, F., Scalaidhe, S., \& Goldman-Rakic, P. (1993). Dissociation of object and spatial processing domains in primate prefrontal cortex. Science, 260, 1955-1958.

Wilson, H., \& Cowan, J. (1972). Excitatory and inhibitory interactions in localized populations of model neurons. Biophys. J., 12, 1-24.

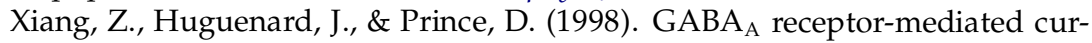
rents in interneurons and pyramidal cells of rat visual cortex. J. Physiol., 506, 715-730.

Received July 17, 2003; accepted November 21, 2003. 